\title{
3 Research Square

\section{IL-6 knockout alleviates inflammatory response and improve acute lung injury via regulating macrophage polarization in sepsis}

\section{Jinxin Zhang}

Fourth Military Medical University: Air Force Medical University

\section{Kuo Shen}

Fourth Military Medical University: Air Force Medical University

Jiangang Xie

Fourth Military Medical University: Air Force Medical University

Shanshou Liu

Fourth Military Medical University: Air Force Medical University

Xiaozhi Bai

Fourth Military Medical University: Air Force Medical University

Jing Wang

Fourth Military Medical University: Air Force Medical University

Ming Zhao

Fourth Military Medical University: Air Force Medical University

Jin Li

Fourth Military Medical University: Air Force Medical University

Shaohui Li

Fourth Military Medical University: Air Force Medical University Junjie Li

Fourth Military Medical University: Air Force Medical University Wen Yin ( $\square$ xjyyyw@126.com )

Fourth Military Medical University: Air Force Medical University https://orcid.org/0000-0002-8108-1951

\section{Research}

Keywords: Macrophage, Sepsis, Inflammation, IL-6

Posted Date: June 15th, 2021

DOI: https://doi.org/10.21203/rs.3.rs-618570/v1 
License: (c) (i) This work is licensed under a Creative Commons Attribution 4.0 International License. Read Full License 


\section{Abstract}

Background Sepsis is a fatal disease with a high rate of morbidity and mortality, during which acute lung injury is the earliest and most serious complication. Macrophage plays a crucial role in the initiation and progress of sepsis. This study meant to explore the effect of IL-6 knockout in CLP induced sepsis.

Methods In this study, cecal ligation and puncture (CLP) was performed on wildtype and interleukin 6 (IL6) knockout $\mathrm{C} 57$ mice. General condition and death rate of sepsis mice were observed. organ samples (lungs, livers, kidneys and hearts) and serum were collected for histology observation and inflammatory cytokine detection. Lung tissue injury detection were conducted via lung injury score, wet/dry ration and protein concentrations measurement of Bronchoalveolar lavage fluid (BALF). In in vivo studies, RAW264.7 macrophages were transfected with IL-6 specific siRNA and treated with LPS. After exposed to IL-6 specific siRNA and LPS, expression of inflammatory cytokines interleukin 1 (IL-1), tumor necrosis factor-a (TNF-a), IL-6 and interleukin 10 (IL-10) were detected by RT-qPCR, concentration of IL-1 and TNF-a in culture supernatant were detected by ELISA and M1 and M2 markers were detected by western blot and flow cytometry.

Results We constructed CLP induced sepsis models and found that inhibition of IL-6 could improve general condition and death rate of sepsis mice. Mice in IL-6 knockout group display improved tissue damage, especially in the lung tissue. IL- 6 knockout relieved inflammatory cytokines storm in both serum and bronchoalveolar lavage fluid while polarized macrophage to an anti-inflammatory M2 phenotype. In cell model, inhibition of IL- 6 could alleviate LPS induced expression of inflammatory cytokines IL-1, TNF$a$, and IL- 6 in macrophages. Western blot and Flow cytometry results indicated that expression of M1 markers (iNOS and CD86) in LPS stimulated macrophages were significantly declined while M2 (Arg-1 and CD206) were enhanced when expression of IL-6 was blocked.

Conclusion Inhibition of IL-6 alleviated LPS induced inflammation and exerted protective effect in sepsis via regulating macrophage function and polarization.

\section{Background}

Sepsis, a life-threatening organ dysfunction induced by a dysregulated host response to infection, is a common disease in the Intensive Care Unit (ICU) [1, 2]. With a high rate of morbidity and mortality, sepsis features complex pathogenesis and serious condition, which is closely associated with multiple organ dysfunction syndrome (MODS) and even death[3, 4]. Among all the complications of sepsis, acute lung injury is the earliest and most severe one for abundant blood flow and vulnerable structure[2, 5]. During the early stages of sepsis, excessive inflammatory factors and reactive oxygen species would damage pulmonary capillary endothelium and increase vascular permeability, leading to inflammatory cell infiltration and further damage[3, 6]. Despite the progresses in diagnostic approach and supportive treatment, sepsis accounts for nearly 40\% death in ICU[7]. In US, sepsis affects more than 1.7 million people every year, of which approximately 270,000 dies[2, 8]. Besides, sepsis put great economic burden 
on both patients and health system. The total medical cost for sepsis is as high as 24 billion U.S. dollars, and the number continues to climb year by year $[1,8]$.

Emerging studies indicated that macrophages play a crucial role in occurrence and development of sepsis by initiating the inflammation cascade reaction $[4,6]$. Macrophages can be divided into two categories roughly: classically activated macrophages (M1) and alternatively activated macrophages (M2) $[9,10]$. M1 macrophages are involved in resistance to pathogens by releasing pro-inflammatory cytokines, while M2 polarized macrophages help to repair and remodel tissue, as well as resisting parasites and tumor growth[10,11]. M1 macrophages can recognize invading pathogens signals and induce the expression of wide varieties of inflammatory mediators, including TNF-a, IL-1, IL- 6 and reactive oxygen species (ROS) $[12,13]$. There is no doubt that these mediators are helpful for elimination of pathogen, but excessive inflammatory cytokines and ROS lead to tissue damage[14]. Consequently, inhibition of M1 polarization of macrophages downregulation of inflammatory cytokines secretion is a therapeutic target of sepsis[15].

IL- 6 is one of the most important proinflammatory cytokines secreted by M1 macrophages in early stage of cytokine storm[16]. Researches indicated that IL-6 level is closely related to progression and prognosis of sepsis, organ dysfunction and death[17]. Blood IL-6 level in most sepsis patients increased significantly at early stage[18, 19]. Hence, IL-6 has been regarded as a biomarker in clinic to anticipate and monitor state of sepsis[20]. However, how IL-6 exerts effects on the progression of sepsis remains unclear. So far conflicting data on the blockage of IL-6 in animal models of sepsis were reported. In the present study, we explored the role of IL-6 in sepsis by regulating macrophage polarization in vivo and in vitro. These data confirmed that IL-6 knockout can attenuate lung inflammation and promote macrophages toward M2 types. Such findings may offer a new way on the treatment of sepsis.

\section{Materials And Methods 2.1 Animals}

Healthy adult C57/BL6 mice were brought from Experimental Animal Centre of Fourth Military Medical University (Shaanxi, China). Mice were raised under pathogen-free conditions at room temperature (22$24^{\circ} \mathrm{C}$ ) in a $12 \mathrm{~h}$ light $/ 12 \mathrm{~h}$ dark cycle. Animal care and experimental protocols were approved by Animal Experimental Committee of Fourth Military Medical University.

\subsection{Animal model}

Cecal ligation and puncture (CLP) was conducted to induce sepsis as previously described[12]. 24 WT C57 mice (12 for CLP group and 12 for sham group) and 12 IL 6 knock out mice went through the process of CLP. Briefly, mice were anesthetized with isoflurane before operation. The ligated part of the cecum was punctured through with a 21-gauge needle. After repositioning of the bowel, the abdomen was closed layer by layer using a 4.0 surgical suture and metallic clips. Sham animals underwent the same procedure without ligation and puncture of the cecum. Clinical disease score (CDS) (Table.1) and 
muridae sepsis score (MSS) (Table.2) were conducted on CLP induced sepsis mice. 24h after operation, the mice were sacrificed at different time points to obtain serum, lung, liver, heart, and kidney samples for inflammatory cytokines measurement and histological observation.

\subsection{Histology and tissue processing}

Mice were sacrificed at different time points $(0,6,12,24 \mathrm{~h})$ after operation, and organ samples (lungs, livers, kidneys and hearts) were collected, fixed in $10 \%$ buffer formalin for $24 \mathrm{~h}$ and embedded in paraffin using standard procedures. Embedded samples were sectioned and stained with hematoxylin and eosin for morphological analysis.

\subsection{Lung injury measurement}

The entire lungs were collected for lung wet/dry ratio (W/D) measurement in order to assess pulmonary edema after the mice were executed in 0,6,12, $24 \mathrm{~h}$. Bronchoalveolar lavage was performed as following steps. The lungs were injected with $0.9 \%$ saline $(0.5 \mathrm{~mL})$ through the main bronchus and repeated for three times. The BALF was centrifuged for $10 \mathrm{~min}$ at $1200 \mathrm{rpm}$. The supernatants were collected and stored at $-80^{\circ} \mathrm{C}$. Protein concentration in BALF was determined by a protein detection kit.

\section{$2.5 \mathrm{Immunofluorescence} \mathrm{staining}$}

Lung, liver and heart infiltrating inflammatory cells were detected by DAPI (Bio-rad), F4/80 (Bio-rad), iNOS (Bio-rad) and CD206(Bio-rad) monoclonal mouse antibodies according to manufacturer's instructions. Immunofluorescence signals were visualized with secondary antibodies by FSX100 microscope (Olympus).

\subsection{Real-time quantitative RT-PCR}

Livers from mice were obtained $12 \mathrm{~h}$ after induction of CLP and prepared as described above. The total RNA was extracted with the TRIZOL agent (Takara) according to the manufacturer's instructions. cDNA were synthesized with PrimeScript ${ }^{\text {TM }}$ RT reagent Kit (Takara). The RT-qPCR was performed using SYBR Green (Takara)in the CFX Connect (BIO-RAD), using $\beta$-Actin as internal control. The thermal cycler procedure was used: $95^{\circ} \mathrm{C}$ for $30 \mathrm{~s}$, followed by 40 cycles $5 \mathrm{~s}$ at $95^{\circ} \mathrm{C}$ followed by $34 \mathrm{~s}$ at $60^{\circ} \mathrm{C}$. The primers used for RT-qPCR were following: IL-1 $\beta-F\left(5^{\prime}-C C C T G A A C T C A A C T G T G A A A T A G C A-3^{\prime}\right)$ / R(5'-CCCAAGTCAAGGGCTTGGAA-3'), IL-6-F(5'-GGGACTGATGCTGGTGACAA-3')/R(5'TCCACGATTTCCCAGAGAACA-3'), TNF-aF(5'-ATACACTGGCCCGAGGGAAC-3')/R(5'-CCACATCTCGGATCATGCTTTC-3'), IL- 10F(5'-GATAGAGCGCAACAAGCAGAA-3')/ R(5'-CAGTGAGGCCCATACCAGAA-3'), $\beta$-Actin F(5'-GTACGCCAACACAGTGCTG-3')/R 5'-CGTCATACTCCTGCTTGCTG-3').

\subsection{ELISA}

IL-1 $\beta, I L-6, I L-10$ and TNF- $\alpha$ concentrations from mice serum were detected by enzyme linked immunosorbent assay (ELISA) kits (Boster, China) according to the manufactures' instructions.

\subsection{Flow cytometry assay}


Cells collected from BAL fluid were detected by flow cytometry. First, cells were collected from BAL fluid by centrifuge at $300 \mathrm{~g}$ for 10 minutes and then incubated with anti-mouse F4/80, CD206, and CD86 antibody (BD, USA) for 30 minutes at $4{ }^{\circ} \mathrm{C}$. Then, cells were analyzed on a flow cytometer (BD, USA) and data were analyzed with FlowJo software.

\subsection{Western blot analysis}

Protein samples from lung tissue were extracted by RIPA lysis buffer, and the concentration of which were detected by BCA kit. The protein samples were separated by electrophoresis in a $10 \%$ polyacrylamide gel and transferred onto PVDF membranes. Then the membranes were blocked in $5 \%$ non-fat dried milk with Tris-buffered solution containing $0.1 \%$ Tween-20 (TBST) at room temperature for $1 \mathrm{~h}$ and incubated overnight at $4{ }^{\circ} \mathrm{C}$ with Arg- 1 and iNOS primary antibody (CST, USA). Then, membranes were incubated for $1 \mathrm{~h}$ with secondary antibody (Boster, China). The results were visualized with ECL on Tanon 5500 Imaging System (Shanghai, China). ImageJ was used to quantify densities of the bands, while GraphPad Prism was used for graphs.

\subsection{Statistical analysis}

The data sets used one-way ANOVA and then compared the mean groups with the student-Newman Coles multiple comparison test. The proportional-risk model was used for statistical analysis of survival studies. $\mathrm{P}<0.05$ was considered significant.

\section{Results}

\subsection{IL-6 Knockout (KO) improved the survival rates of the septic mice.}

CLP were conducted on 20 WT C57 mice (10 for CLP group and 10 for sham group) and 10 IL-6 knockout mice. CDS was applied for evaluation of septic mice at 24 and $48 \mathrm{~h}$ after CLP. In both $24 \mathrm{~h}$ and $48 \mathrm{~h}$ following CLP, the CDS was significantly higher in septic animals compared to sham group (Fig. 1A), indicating that we successfully constructed the septic model. MSS of IL-6 KO sepsis mice is lower than that of WT group (Fig. 1B). Meanwhile, the survival rates were observed in the consecutive $72 \mathrm{~h}$ after operation. Results indicated that WT mice in CLP group died more than half $(6 / 10)$ at $24 \mathrm{~h}$ after operation. While in IL-6 KO group, the death rate was significantly decreased $(2 / 10)$ compared with CLP groups. And at 48h after operation, 8/10 WT mice in CLP group died, while 4/10 IL-6 KO mice in CLP group died. This result indicated that knockout of IL- 6 in CLP mice could significantly improve the survival rates $(p<0.05)$ (Fig. 1C).

\subsection{IL-6 Knockout alleviated lung lesion and dysfunction.}

$24 \mathrm{~h}$ after operation, mice in different group were sacrificed for lung injury assessment. Lung tissue were collected for histological detection, while lung injury score, lung tissue wet/dry ratio and protein 
concentration of BALF detection were conducted for lung injury assessment. As indicated in Fig. 2A, lung tissues in $\mathrm{LL}-6^{+/+}+\mathrm{CLP}$ mice displayed obvious inflammatory infiltration, interstitial edema and alveolar structural damages, while those phenomena were remarkedly improved in the IL- $6^{-/}+\mathrm{CLP}$ group. Compared with IL- $-6^{+/+}+$CLP group, the lung injury score and wet/dry ration of IL- $6^{-/-}+$CLP group were significantly decreased (Fig. 2B and C). In addition to this, protein concentrations of BALF in IL- $6^{-/-}+$CLP group also declined significantly at 12 and $24 \mathrm{~h}$ after operation than IL- $6^{+/+}+$CLP group (Fig. 2D).

\subsection{IL-6 knockout mitigate CLP induced inflammatory cytokine storm.}

Serum and BALF were collected from mice in different treatment groups for inflammatory cytokine detection by ELISA. As is shown in Fig. 3A, inflammatory cytokines (IL-1 $\beta$, TNF-a, and IL-6) expression in $\mathrm{IL}-6^{+/+}+\mathrm{CLP}$ mice were significantly increased in serum than that of sham group. While in IL- $6^{-/-}$mice, concentrations of these inflammatory cytokines were all decreased significantly than IL- $6^{+/+}+$CLP group. Consistent with this, same phenomena were observed in the BALF. In lung tissues, CD206 and iNOS immunofluorescence staining were performed to observe macrophage infiltration. As is shown in Fig. 3C, number of $\mathrm{iNOS}^{+}(\mathrm{M} 1)$ macrophages in IL- $6^{+/+}+$CLP was much larger than that of sham group. And macrophage M1 polarization of IL-6 KO mice were reduced compared with WT mice. In the meantime, M2 polarization of IL- $6^{-/-}+$CLP group was increased than that of IL- $6^{+/+}+$CLP. Hence, aforementioned results suggested that IL- 6 knockout distinctly prevented the upregulation of pro-inflammatory cytokines and promoted anti-inflammatory trait.

\subsection{IL-6 interference alleviated macrophage M1 polarization and promotes $\mathrm{M} 2$ polarization.}

In in vivo studies, $1 \mu \mathrm{g} / \mathrm{ml}$ LPS and IL-6 specific siRNA were used to stimulate RAW264.7 macrophages. Inflammatory cytokines IL-1 $\beta$, IL-6, TNF- $\alpha$ and IL-10 were detected by RT-qPCR. The mRNA levels of these inflammatory cytokines were significantly increased by LPS stimulation. To investigate the role of IL-6 in inflammatory response, we constructed IL- 6 specific siRNA. After transfection for 24 h, IL-6 expression was successfully decreased at both the transcriptional and translational levels (Fig. 4A). Hence, we treated siRNA transfected RAW264.7 cells with LPS. ELISA detection revealed the increase of TNF- $a$, IL-1 $\beta$ concentration in culture supernatant when IL-6 was inhibited (Fig. 5B). RT-qPCR results indicated that LPS induced up-regulation of inflammatory cytokines TNF- $\alpha$, IL-1 $\beta$ and IL- 6 were ameliorated by IL- 6 interference (Fig. 5C), while expression of IL-10 was increased. And as showed in Fig. 4D, when IL-6 was silenced by siRNA, iNOS protein expression was overwhelmingly increased, while Arg-1 was decreased. Meanwhile, flow cytometry indicated that when IL- 6 was inhibited, expression of M1 macrophage (CD86 ${ }^{+}$ /CD206 ${ }^{-}$) was decreased while M2 (CD86 $/ 6^{-}$CD206 $\left.{ }^{+}\right)$was increased (Fig. 5E).

\section{Discussion}


Sepsis is characterized by systemic inflammatory response syndrome which often associated with MODS and even death[21]. Besides, the morbidity and mortality rates of sepsis remained stubbornly high despite the progresses in diagnostic approach and supportive treatment[2,3]. Consequently, it is urgent to discover effective therapeutic targets and intervening measures. Wide arrays of studies confirm that macrophages play an important role in sepsis and other diseases via mediating inflammatory producers[22, 23]. Macrophages can be classified as 2 phenotypes: classically activated macrophages (M1) which contribute to tissue injury by producing excessive reactive cytotoxic oxidants to destroy pathogens and alternatively activated macrophages (M2) which suppress inflammation and promote repair by phagocytizing dead neutrophils and synthesizing molecules $[9,10]$. At the beginning of inflammatory response phase, macrophages are activated by invading pathogens and other harmful substance, leading to excessive inflammatory cytokine storm[22, 24]. These inflammatory cytokines are undoubtfully helpful in host defense and eliminating pathogens, while these overzealous productions can be deleterious, leading to a "double-edge sword" property reducing pathogens at the expense of organ injury[6]. Based on understanding of molecular mechanisms underlying sepsis, novel therapies have been designed to suppress macrophage induced cytokine storm and transform macrophages from M1 to M2[12]. In in vivo studies, we revealed that interference of IL- 6 would alleviate LPS induced inflammatory response. When expression of IL- 6 was inhibited, expression of inflammatory cytokines,such as IL-1 $\beta$ and TNF-a $\square$ were significantly decreased, and ratio of M1 macrophage declined while M2 increased.

IL-6 plays vital roles in host defense against acute infections via activating acute-phase reactions and immune responses[20]. In sepsis and other infected lesions, IL-6 is produced by monocytes and macrophages when stimulated with bacterial, viral or fungal components such as lipopolysaccharide and peptidoglycan with PAMPs[25]. IL-6, as a soluble mediator, sends out inflammatory signals from localized lesions to the whole body and provides an SOS signal to trigger the host defense against emergent events such as infection and trauma[19]. Concentration of IL-6 in blood as a prognostic factor has been evaluated in several studies. And results showed that IL-6 has a high sensitivity and specificity for the detection of sepsis or early sepsis[4,26]. In this study, we constructed sepsis models via Cecal ligation and puncture (CLP), and observed inflammatory response and tissue damage in lung tissue of WT and IL$6 \mathrm{KO}$ mice. Results indicated that when expression of IL-6 were inhibited, expressions of inflammatory cytokines in CLP induced sepsis mice were decreased and the Muridae Sepsis Score were decreased. 24 $\mathrm{h}$ after operation, the damage of tissues, particularly the lung, was significantly improved, and the death rate during $48 \mathrm{~h}$ after operation was declined in IL-6 KO mice than that of WT. IL-6 is an important inflammatory mediator which plays a crucial role in inducing inflammatory cascade reaction via several signal pathways, including JAK1/STAT3 pathway[27]. When expression of IL-6 was inhibited, CLP induced inflammatory response and organ damage were improved.

Lung is one of the most easily affected organs by sepsis[28]. In sepsis patients, acute lung injury and acute respiratory distress syndrome (ARDS) are the most common and serious complication, the incidence of which is as high as $40 \%$ and the mortality is about $40-50 \%[2,29]$. Sepsis-induced ARDS refers to damage of alveolar epithelial cells and capillary endothelial cells caused by excessive inflammatory cytokines and ROS, which leads to pulmonary edema, diffused interstitial and alveolar 
damage[30]. The occurrence and development of ARDS are closely associated with pulmonary edema caused by endothelial damage and increased vascular permeability[31, 32]. Integrality of alveolar barrier is essential to prevent pulmonary edema and promote ARDS relief[33]. Therefore, vascular permeability of alveolar epithelium is an important indicator of occurrence and severity of ARDS. In this study, CLP induced sepsis resulted in pulmonary edema, inflammatory cell infiltration and alveolar damage. But in IL-6 KO sepsis mice, above lung injury manifestation was remarkably improved. And lung injury score and wet/dry weight ratio indicated that lung injury especially vascular permeability was mitigated. And when expression of IL-6 was suppressed, infiltration of inflammatory cell, especially M1 macrophage, was decreased CLP induced sepsis mice. Consequently, aforementioned results indicated that when expression of IL- 6 was blocked, the inflammatory response of macrophage in CLP induced sepsis was decreased, tissue damage was improved and survival rate of mice was increased.

\section{Conclusion}

In this study, we found that inhibition of IL-6 could mitigate LPS induced inflammatory cytokines storm in macrophages and polarize macrophages to anti-inflammatory M2 phenotype. And in CLP induced sepsis model, knockout of IL-6 could relieve the inflammation cytokines storm in serum and BALF, reduce infiltration of inflammatory macrophage and improve lung tissue damage in lung which increase survival rate of mice. Collectively, we illustrated a novel potentially therapeutic mechanism that inhibition of IL-6 could alleviate the LPS induced inflammatory cytokines and increase ratio of M2 macrophages, hence improve the inflammatory reaction and the lung injury in sepsis.

\section{Abbreviations}

CLP

cecal ligation and puncture;

IL-6

interleukin 6;

BALF

bronchoalveolar lavage fluid;

IL-1

interleukin 1;

TNF-a

tumor necrosis factor-a;

IL-10

interleukin 10;

ICU

intensive care unit;

MODS

multiple organ dysfunction syndrome; 
ROS

reactive oxygen species;

CDS

clinical disease score;

MSS

muridae sepsis score;

KO

knockout;

ARDS

acute respiratory distress syndrome.

\section{Declarations}

\section{Acknowledgments}

The authors thank all the other members in their laboratory for their insight and technical support.

\section{Author contributions}

Conceptualization, Wen Yin and Junjie Li.

Methodology (animal model), Jinxin Zhang and Jin Li;

Investigation, Kuo Shen and Xiaozhi Bai;

Validation, Shaohui Liu;

Data Curation, Shanshou Liu and Jin Li;

Writing - Original Draft Preparation, Jinxin Zhang and Kuo Shen;

Writing - Review \& Editing, Wen Yin and Junjie Li;

Visualization, Jin Wang and Ming Zhao;

Supervision, Junjie Li;

Project Administration Wen Yin;

Funding Acquisition, Wen Yin.

\section{Conflicts of interest}

No potential conflicts of interest were disclosed.

\section{Funding}


This work was supported by grants from the National Natural Science Foundation of China (81871587 and 81530064).

\section{Ethics approval}

All animal studies were approved by the Animal Care and Use Committee of Fourth Military Medical University, and all experimental protocols were approved by the Medical and Ethics Committee of Xijing Hospital, Fourth Military Medical University, Xian, China.

\section{Data availability statement}

The data used to support the findings of this study are available from the corresponding author upon request.

\section{References}

1. Rudd KE, et al. Global, regional, and national sepsis incidence and mortality, 1990-2017: analysis for the Global Burden of Disease Study. Lancet. 2020;395(10219):200-11.

2. Bauer $M$, et al. Mortality in sepsis and septic shock in Europe, North America and Australia between 2009 and 2019- results from a systematic review and meta-analysis. Crit Care. 2020;24(1):239.

3. Font MD, Thyagarajan B, Khanna AK. Sepsis and Septic Shock - Basics of diagnosis, pathophysiology and clinical decision making. Med Clin North Am. 2020;104(4):573-85.

4. Cecconi M, et al. Sepsis and septic shock. Lancet. 2018;392(10141):75-87.

5. Surviving Sepsis Campaign International Guidelines. Pediatrics, 2020. 145(5).

6. Hori H, Kim Y. Inflammation and post-traumatic stress disorder. Psychiatry Clin Neurosci. 2019;73(4):143-53.

7. Luhr R, et al. Trends in sepsis mortality over time in randomised sepsis trials: a systematic literature review and meta-analysis of mortality in the control arm, 2002-2016. Crit Care. 2019;23(1):241.

8. Rhee C, et al. Incidence and Trends of Sepsis in US Hospitals Using Clinical vs Claims Data, 20092014. Jama. 2017;318(13):1241-9.

9. Locati M, Curtale G, Mantovani A. Diversity, Mechanisms, and Significance of Macrophage Plasticity. Annu Rev Pathol. 2020;15:123-47.

10. Orecchioni M, et al. Macrophage Polarization: Different Gene Signatures in M1(LPS+) vs. Classically and M2(LPS-) vs. Alternatively Activated Macrophages. Front Immunol. 2019;10:1084.

11. Atri C, Guerfali FZ, Laouini D. Role of Human Macrophage Polarization in Inflammation during Infectious Diseases. Int J Mol Sci, 2018. 19(6).

12. Shen $\mathrm{K}$, et al. Exosomes from adipose-derived stem cells alleviate the inflammation and oxidative stress via regulating Nrf2/HO-1 axis in macrophages. Free Radic Biol Med. 2021;165:54-66. 
13. Minton K. Immunometabolism: Stress-induced macrophage polarization. Nat Rev Immunol. 2017;17(5):277.

14. Gu Z, et al. Mechanism of Iron Oxide-Induced Macrophage Activation: The Impact of Composition and the Underlying Signaling Pathway. J Am Chem Soc. 2019;141(15):6122-6.

15. Venet $F$, Monneret $G$. Advances in the understanding and treatment of sepsis-induced immunosuppression. Nat Rev Nephrol. 2018;14(2):121-37.

16. Inoue KI, Sagawa T, Takano H. Role of IL-6 in Severe Inflammation. Am J Respir Crit Care Med. 2021;203(1):140-1.

17. Díaz-Bulnes P, et al. Crosstalk Between Hypoxia and ER Stress Response: A Key Regulator of Macrophage Polarization. Front Immunol. 2019;10:2951.

18. Kang S, et al., Historical overview of the interleukin-6 family cytokine. J Exp Med, 2020. 217(5).

19. Rose-John S. The Soluble Interleukin 6 Receptor: Advanced Therapeutic Options in Inflammation. Clin Pharmacol Ther. 2017;102(4):591-8.

20. Uciechowski P, Dempke WCM. Interleukin-6: A Masterplayer in the Cytokine Network. Oncology. 2020;98(3):131-7.

21. Napolitano LM. Sepsis 2018: Definitions and Guideline Changes. Surg Infect (Larchmt). 2018;19(2):117-25.

22. Viola A, et al. The Metabolic Signature of Macrophage Responses. Front Immunol. 2019;10:1462.

23. Wynn TA, Vannella KM. Macrophages in Tissue Repair, Regeneration, and Fibrosis. Immunity. 2016;44(3):450-62.

24. Stanski NL, Wong HR. Prognostic and predictive enrichment in sepsis. Nat Rev Nephrol. 2020;16(1):20-31.

25. Rossi JF, et al. Interleukin-6 as a therapeutic target. Clin Cancer Res. 2015;21(6):1248-57.

26. Unver N, McAllister F. IL-6 family cytokines: Key inflammatory mediators as biomarkers and potential therapeutic targets. Cytokine Growth Factor Rev. 2018;41:10-7.

27. Murray PJ. The JAK-STAT signaling pathway: input and output integration. J Immunol. 2007;178(5):2623-9.

28. Park I, et al., Neutrophils disturb pulmonary microcirculation in sepsis-induced acute lung injury. Eur Respir J, 2019. 53(3).

29. Genga KR, Russell JA. Update of Sepsis in the Intensive Care Unit. J Innate Immun. 2017;9(5):44155.

30. Kumar V. Pulmonary Innate Immune Response Determines the Outcome of Inflammation During Pneumonia and Sepsis-Associated Acute Lung Injury. Front Immunol. 2020;11:1722.

31. Moussa MD, et al. Evaluation of endothelial damage in sepsis-related ARDS using circulating endothelial cells. Intensive Care Med. 2015;41(2):231-8.

32. Vassiliou AG, et al., Endothelial Damage in Acute Respiratory Distress Syndrome. Int J Mol Sci, 2020. 21(22). 
33. Sinha P, et al. Latent class analysis of ARDS subphenotypes: a secondary analysis of the statins for acutely injured lungs from sepsis (SAILS) study. Intensive Care Med. 2018;44(11):1859-69.

\section{Tables}

Table 1

The clinical disease (CDS) was applied for evaluation of septic mice in the article

\begin{tabular}{|ll|}
\hline Sign & Score \\
\hline Piloerection (not present-extensive) & $0-2$ \\
\hline Conjunctivitis (not present-bilateral) & $0-2$ \\
\hline Grooming behavior (normal-none) & $0-2$ \\
\hline Mobility (normal-reduced-immobile) & $0-3$ \\
\hline Signs of peritoneal irritation (none-tiptoeing and broad pace) & $0-2$ \\
\hline Position of the ears(erect-flat) & $0-1$ \\
\hline Stool consistency (normal-sticky or diarrhea) & $0-1$ \\
\hline Anemic appearance (absent or present) & $0-1$ \\
\hline Moribund & $0-1$ \\
\hline Total Clinical Disease Score (no signs of disease-maximum) & $0-15$ \\
\hline
\end{tabular}

Table 2

Murine sepsis score (MSS) was applied for evaluation of septic mice in the article

\begin{tabular}{|c|c|c|c|c|}
\hline Score & 0 & 1 & 2 & 3 \\
\hline Appearance & Smooth & slightly ruffled fur & $\begin{array}{l}\text { Majority of fur on back } \\
\text { is ruffled }\end{array}$ & $\begin{array}{l}\text { Piloerection, puffy } \\
\text { appearance }\end{array}$ \\
\hline $\begin{array}{l}\text { Level of } \\
\text { consciousness }\end{array}$ & Active & $\begin{array}{l}\text { Active, avoids } \\
\text { standing upright }\end{array}$ & $\begin{array}{l}\text { Active only when } \\
\text { provoked }\end{array}$ & $\begin{array}{l}\text { Non-responsive, } \\
\text { even when } \\
\text { provoked }\end{array}$ \\
\hline Activity & Normal & $\begin{array}{l}\text { Suppressed eating, } \\
\text { drinking, or running }\end{array}$ & Stationary & $\begin{array}{l}\text { Stationary, even } \\
\text { when provoked }\end{array}$ \\
\hline $\begin{array}{l}\text { Response to } \\
\text { stimulus }\end{array}$ & Normal & $\begin{array}{l}\text { Slowed response to } \\
\text { auditory or touch } \\
\text { stimuli }\end{array}$ & $\begin{array}{l}\text { No response to auditory, } \\
\text { slowed response to } \\
\text { touch }\end{array}$ & $\begin{array}{l}\text { No response to } \\
\text { touch stimuli }\end{array}$ \\
\hline Eyes & Open & $\begin{array}{l}\text { Not fully open, } \\
\text { potentially secretions }\end{array}$ & $\begin{array}{l}\text { Half closed, potential } \\
\text { secretions }\end{array}$ & $\begin{array}{l}\text { Mostly or } \\
\text { completely closed }\end{array}$ \\
\hline $\begin{array}{l}\text { Respiration } \\
\text { quality }\end{array}$ & Normal & $\begin{array}{l}\text { Periods of labored } \\
\text { breathing }\end{array}$ & $\begin{array}{l}\text { Consistently labored } \\
\text { breathing }\end{array}$ & $\begin{array}{l}\text { Labored } \\
\text { breathing with } \\
\text { gasps }\end{array}$ \\
\hline
\end{tabular}



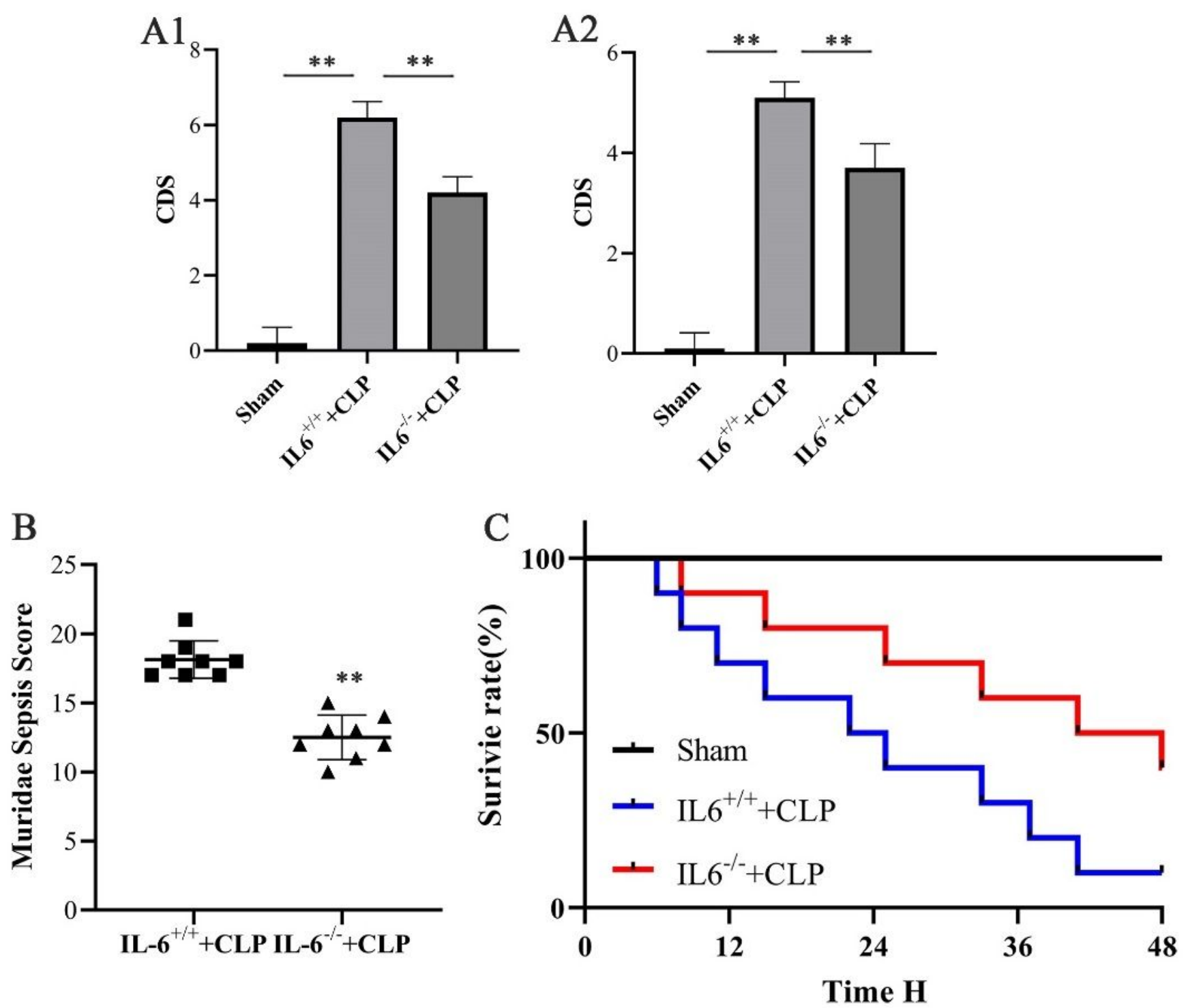

Figure 1

IL-6 Knockout improved the survival rates of the septic mice. A. Clinical disease score evaluation of septic wild type mice at 24(A1) and $48 \mathrm{~h}(\mathrm{~A} 2)$ after CLP, $\star *: P \leq 0.01$. B. Muridae sepsis score of wild type and IL6 knockout sepsis mice, $* *: P \leq 0.01$. C. Survivorship curve of CLP treated wild type and IL-6 KO C57 mice during $72 \mathrm{~h}$ after CLP. 

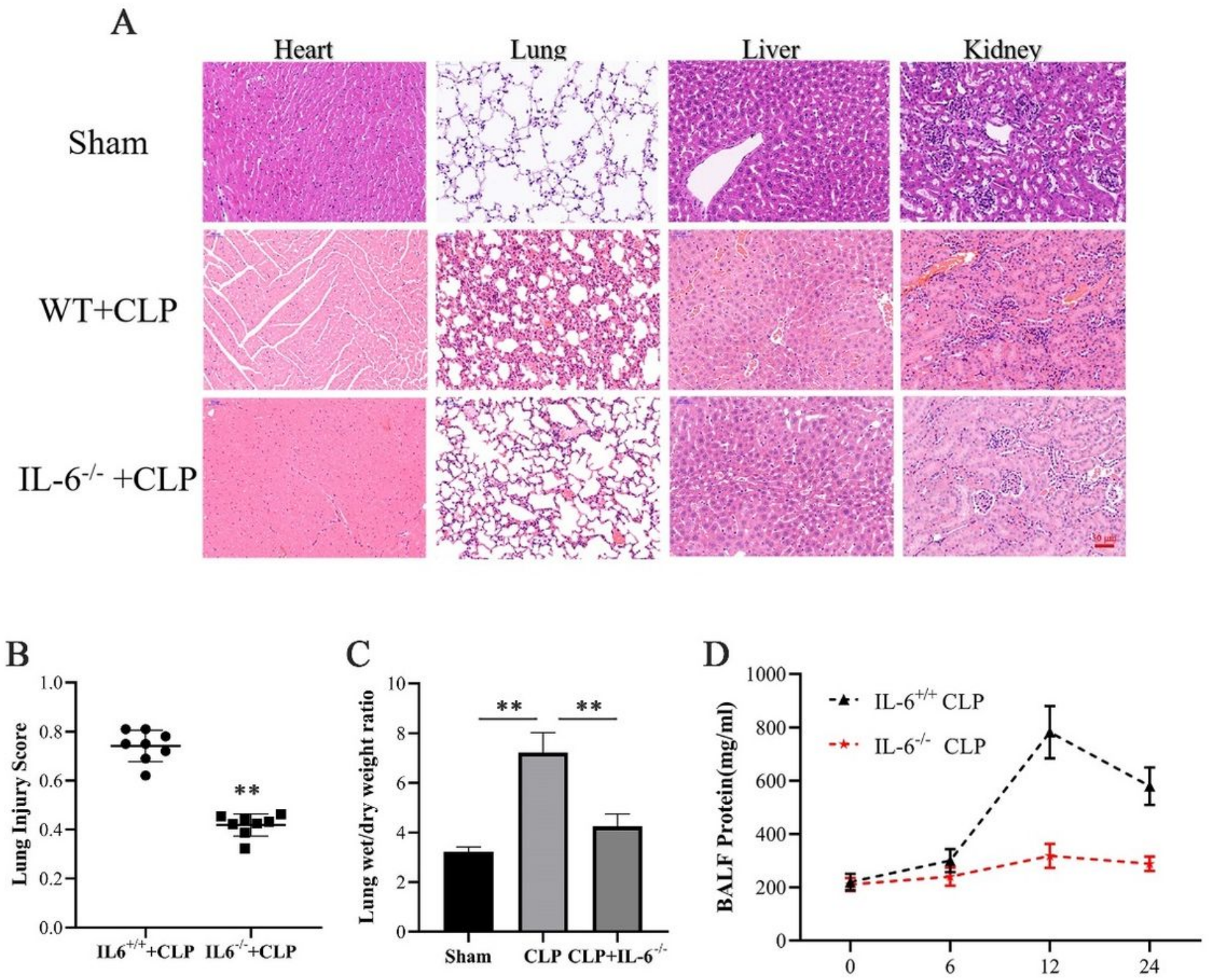

Figure 2

IL-6 Knockout alleviated lung lesion and dysfunction. A. HE results showing histology of the lung, liver, heart, and kidney tissues in CLP treated wild type and IL-6 knockout C57 mice. Scale bar: $50 \mu \mathrm{m}$. B. Lung injury score of CLP treated wild type and IL- 6 knockout C57 mice, $* *: P \leq 0.01$. C. Lung wet/dry ration of CLP treated wild type and IL- 6 knockout C57 mice, $* *$ : $P \leq 0.01$. D. Protein concentrations of BALF in CLP treated wild type and IL-6 knockout C57 mice $24 \mathrm{~h}$ after operation. 

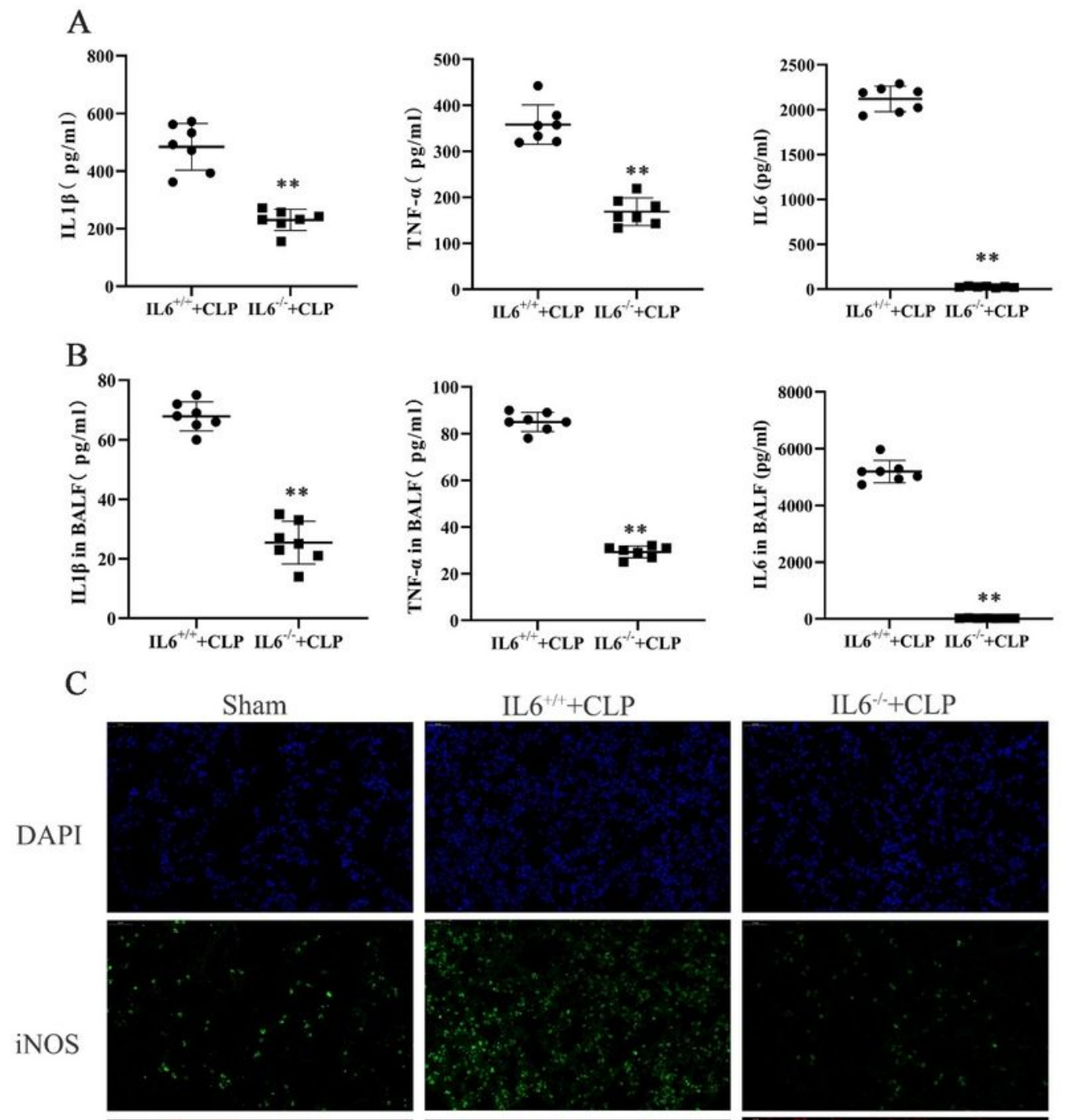

Arg-1
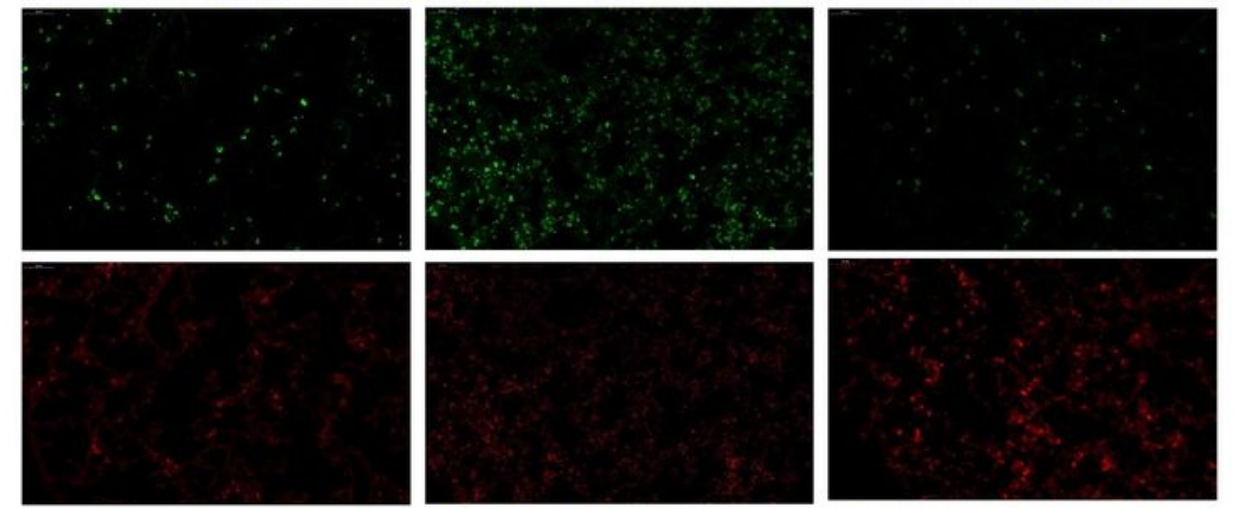

Merge
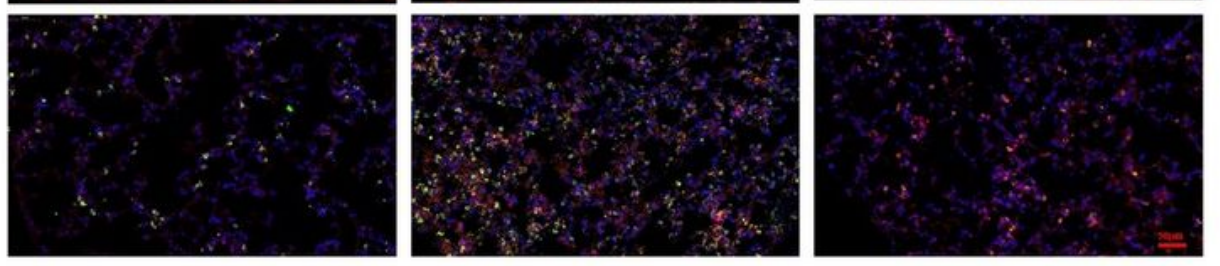

\section{Figure 3}

IL- 6 knockout mitigate CLP induced inflammatory cytokine storm. A. ELISA results of IL-1 $\beta, T N F-a$ and IL6 contents in the mice serum collected from CLP treated wild type and IL- 6 knockout C57 mice. **: P $\leq$ 0.01. C. ELISA results of IL-1 $\beta$, TNF- $\alpha$ and IL- 6 contents in the mice BALF collected from CLP treated wild type and IL- 6 knockout C57 mice. ${ }^{\star}$ : $P \leq 0.01$. iNOS and CD206 expressions in the lung tissues of CLP 
treated wild type and IL-6 knockout C57 mice detected by immunofluorescence. Blue: DAPI; Green: iNOS; Red: CD206. Scale bar: $50 \mu \mathrm{m}$.

A

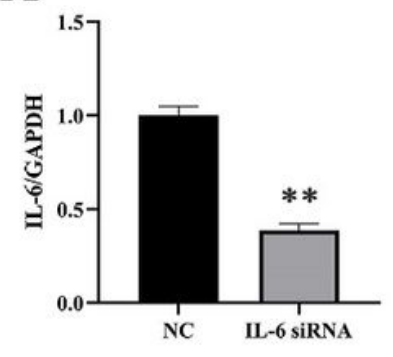

C

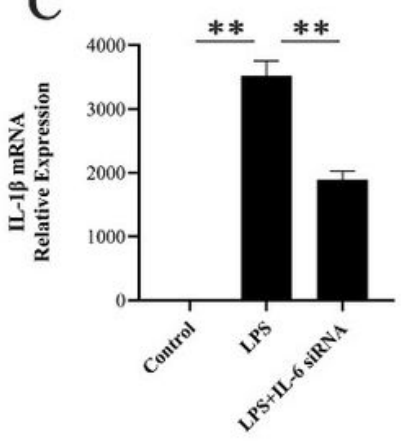

D

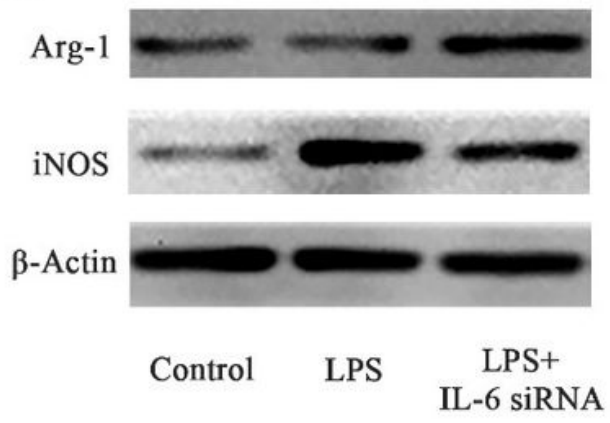

$\mathrm{E}$

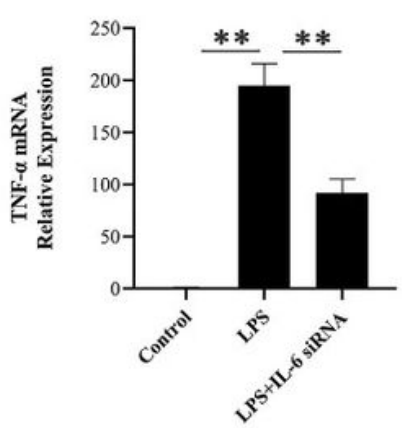

B
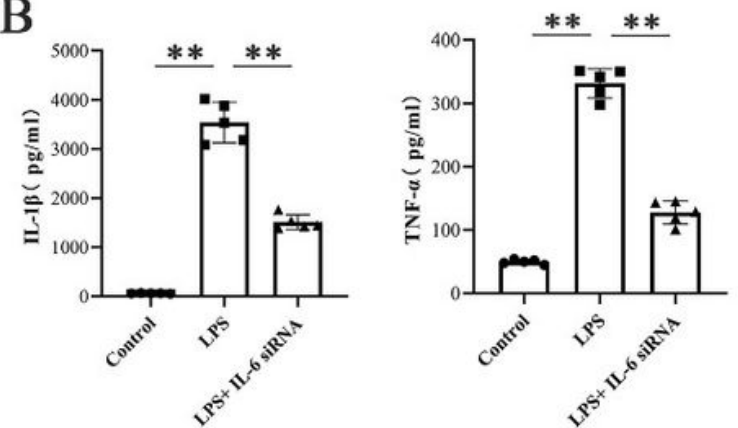

Control IL-6 siRNA
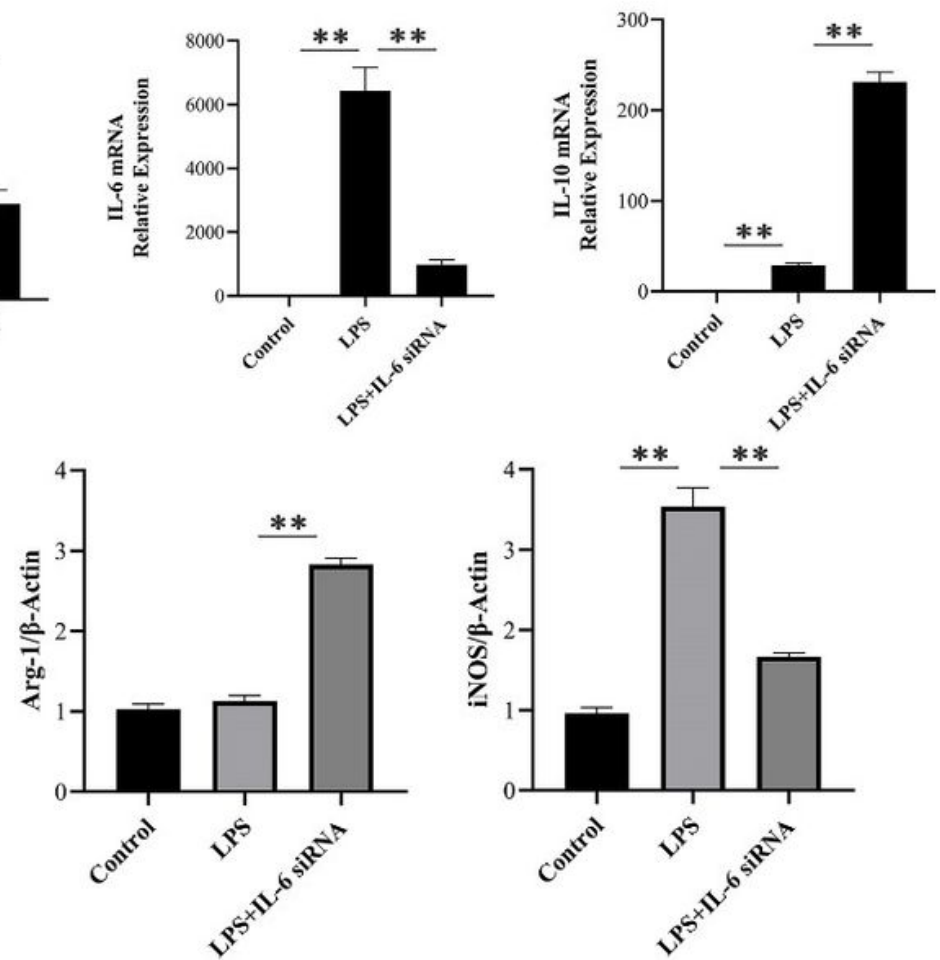
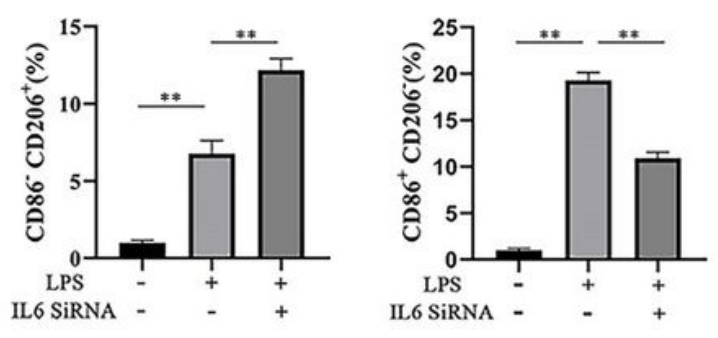

\section{Figure 4}

IL-6 interference alleviated macrophage M1 polarization and promotes M2 polarization. A. IL-6 mRNA and protein expressions detected by RT-qPCR and western blot in RAW 264.7 cells 12 hours after transfected with IL- 6 siRNA. B. ELISA results showing the IL-1 $\beta$ and TNF-a contents in the culture supernatants of RAW264.7 cells 12 hours after different treatments, $* *: P \leq 0.01$. C. RT-qPCR results showing the mRNA 
expressions of inflammatory cytokines (IL-1 $\beta$, TNF-a, IL-6 and IL-10) in RAW264.7 cells 12 hours after different treatments. $\star \star: P \leq 0.01$. D. M1 and M2 macrophage specific maker iNOS and Arg1 protein expressions of RAW264.7 cells 12 hours after different treatments detected by western blot. $* *: P \leq 0.01$. E. Flow cytometry results of CD86 and CD206 expressions in RAW264.7 cells 12 hours after different treatments, $* *: \mathrm{P} \leq 0.01$. 